\title{
Hesperidin administration suppresses the proliferation of lung cancer cells by promoting apoptosis via targeting the miR-132/ZEB2 signalling pathway
}

\author{
SONG TAN ${ }^{*}$, LINGLING DAI*, PENGCHENG TAN, WEI LIU, \\ YUEJUN MU, JINGUO WANG, XIAOMING HUANG and AIHUA HOU \\ Department of Oncology, Yantai Hospital of Traditional Chinese Medicine, Yantai, Shandong 264000, P.R. China
}

Received April 9, 2019; Accepted June 10, 2020

DOI: $10.3892 / \mathrm{ijmm} .2020 .4756$

\begin{abstract}
This aim of the present study was to identify the relationship between hesperidin and microRNA (miR)-132, and to study the role of hesperidin and miR-132 in the pathogenesis of non-small cell lung cancer (NSCLC). Computational analysis and luciferase assays were performed to identify the target of miR-132. Subsequently, reverse transcription-quantitative PCR and western blot assays were used to detect the effect of miR-132 and hesperidin on the expression of haematological and neurological expressed 1 (HN1) and zinc finger E-box binding homeobox 2 (ZEB2). Finally, MTT assays and flow cytometry analysis were used to investigate the effect of hesperidin on cell proliferation and apoptosis. ZEB2 was identified as a target gene of miR-132, and transfection with miR-132 mimic reduced the luciferase activity of the wild-type ZEB2 3'-untranslated region (3'-UTR) but not that of the mutant ZEB2 3'-UTR. By contrast, neither transfection with miR-132 mimic nor hesperidin treatment affected HN1 expression. Furthermore, hesperidin evidently inhibited cell proliferation and promoted apoptosis in a dose-dependent manner. Furthermore, the tumour volume in rats transplanted with NSCLC cells and treated with hesperidin was notably smaller compared with that in rats transplanted with NSCLC cells alone, while treatment with hesperidin significantly reduced the colony formation efficiency of NSCLC cells by increasing miR-132 expression and decreasing ZEB2 expression. To the best of our knowledge, the present study demonstrated for the first time that the administration of hesperidin decreased the expression of ZEB2 by upregulating
\end{abstract}

Correspondence to: Professor Aihua Hou, Department of Oncology, Yantai Hospital of Traditional Chinese Medicine, 39 Xingfu Road, Yantai, Shandong 264000, P.R. China

E-mail: herbctm@126.com

*Contributed equally

Key words: non-small cell lung cancer, hesperidin, microRNA-132, zinc finger E-box binding homeobox 2, apoptosis the expression of miR-132, which in turn promoted apoptosis and inhibited the proliferation of NSCLC cells.

\section{Introduction}

Among all types of cancers worldwide, lung cancer is associated with the highest mortality and morbidity, with more than 1.5 million new cases diagnosed worldwide, as reported in 2014 (1). Approximately 85\% of all lung cancer cases are non-small cell lung cancer (NSCLC) (2). Currently, chemotherapy, radiotherapy and surgical resection remain the primary treatment options for NSCLC $(1,2)$. Despite progress in the development of targeted therapies and the improved efficacy of chemotherapy drugs, the 5-year survival rate of patients with NSCLC remains low $(1,2)$. The low survival rate of patients with NSCLC is due to their high chance of tumour metastasis, which is caused by a number of different factors, such as tumour cell invasion/migration, epithelial-mesenchymal transition (EMT) and angiogenesis (3-8). In addition, activation of the $\mathrm{C}-\mathrm{X}-\mathrm{C}$ chemokine receptor type 4 (CXCR4)/stromal cell-derived factor 1 (SDF1) pathway plays an essential role in the metastasis of $\operatorname{NSCLC}(9,10)$.

Hesperidin (5,7,3-trihydroxy-4-methoxy-flavanone 7-rhamnoglucoside), a type of glycoside flavonoid, has been shown to exert anticancer effects by inhibiting proliferation and promoting apoptosis in numerous types of cancer, including lung cancer and breast cancer (11-13). It has also been reported that hesperidin inhibits the growth of NSCLC cells in vitro by regulating the activation of pathways related to immune responses and apoptosis $(14,15)$. Therefore, hesperidin may be used as a novel anti-proliferative agent in the treatment of NSCLC.

As a type of short non-coding RNA, with a length of $\sim 22$ nucleotides, microRNAs (miRNAs or miRs) can regulate the expression of their target mRNAs by binding to the 3'-untranslated region (3'-UTR) $(16,17)$. As miRNAs act as a negative regulators of gene expression, they serve an essential role in regulating the growth, migration, invasion and metastasis of cancer cells (18-20).

In a previous study, it was shown that miR-132 expression is downregulated in clinical samples and cell lines of metastatic lung cancer, and miR-132 could target the regulator of ETM, 
zinc finger E-box binding homeobox 2 (ZEB2), indicating that miR-132 may act as a tumour suppressor (21). miR-132 can also suppress the metastasis and EMT of NSCLC cells by inhibiting the function of ZEB2. Notably, it was observed that two downstream effectors of ZEB2, vimentin and E-cadherin, are also regulated by miR-132, suggesting that miR-132 may be implicated in the invasion and migration of NSCLC cells by regulating the EMT process (22).

The dysregulated miR-132 expression is thought to play an essential role in the pathogenesis of mental disorders (22). In fact, growing evidence has demonstrated that miR-132 can affect the functions of inflammatory and neurotrophic systems, both of which are implicated in the pathogenesis of depression $(23,24)$. A previous study demonstrated that hesperidin increases miR-132 expression in the prefrontal cortex of lipopolysaccharide-treated mice, suggesting that miR-132 is implicated in the function of hesperidin as an antidepressant (25). Other studies have speculated that the increased expression of miR-132 may protect the brain by stimulating the synthesis of neurotrophin and by blocking the onset of neuroinflammation $(26,27)$.

Yi et al (26) reported that the overexpression of ZEB2 is associated with a poor prognosis of NSCLC and shorter survival. By contrast, the knockdown of ZEB2 increased the sensitivity of cancer cells to chemotherapeutic agents by inducing cell cycle arrest in the $S$ phase and by inducing cell apoptosis. These findings indicated that ZEB2 may act as an oncogene in NSCLC. In another study, the knockdown of ZEB2 inhibited the EMT, and the invasion and migration of NSCLC, which is similar to the effect of miR-200c overexpression (28).

It has been reported that the administration of hesperidin can upregulate the expression of miR-132, which is a potential regulator of haematological and neurological expressed 1 (HN1), TGF- $\beta 1$ and ZEB2 (25,28-30). In addition, HN1, TGF- $\beta 1$ and ZEB2 have been shown to be involved in the pathogenesis of NSCLC (31-34). The present study investigated the effect of hesperidin on the proliferation and apoptosis of NSCLC cells.

\section{Materials and methods}

Animals and treatment. A total of 24 adult Sprague-Dawley (SD) male rats (weight, 328-365 g), which were obtained from the Experimental Animal Centre of Yantai Hospital of Traditional Chinese Medicine, were used in this study. All rats were placed in an environment with 50-60\% humidity and a temperature of $22-24^{\circ} \mathrm{C}$. The rats were given free access to water and food, and a $12 \mathrm{~h}$ light-dark cycle was applied. Following 7 days of environmental adaptation, the rats were randomly assigned into two groups; a negative control (NC) group and a hesperidin group, with 12 rats in each group. In the NC group, the rats were transplanted with NSCLC cells. In the hesperidin group, the rats were transplanted with NSCLC cells and treated with $60 \mathrm{mg} / \mathrm{kg} /$ day hesperidin at the same time to investigate the effect of hesperidin on NSCLC. The NSCLC cells were transplanted into the right lung using a 28G1/2 needle. At the end of the experiment, all rats were sacrificed by sodium pentobarbital $(100 \mathrm{mg} / \mathrm{kg}$ i.p.) and the tumour tissues were isolated and measured. Death was confirmed by combined factors, including lack of pulse, breathing, corneal reflex and response to toe pinch; inability to hear respiratory sounds and heartbeat; and failure of syringe and needle to move after percutaneous cardiac puncture following unconsciousness. The weight of SD male rats at the start of the study was $259 \pm 35$ and $263 \pm 38 \mathrm{~g}$ in the $\mathrm{NC}$ and hesperidin groups, respectively, while the weight of the rats at the end of the study was $342 \pm 52$ and $322 \pm 48 \mathrm{~g}$ in the $\mathrm{NC}$ and hesperidin groups, respectively. The tumour volume was measured using callipers and was calculated as the height $\mathrm{x}$ width $\mathrm{x} 2$. All animal experiments in the present study were approved by the Animal Ethics Committee of Yantai Hospital of Traditional Chinese Medicine.

RNA isolation and reverse transcription-quantitative PCR $(R T-q P C R)$. A TRIzol reagent kit (Invitrogen; Thermo Fisher Scientific, Inc.) was used to extract the total RNA from isolated tissue samples or cultured A549 and H460 cells. The concentration of isolated RNA was measured using a NanoDrop 3000 spectrophotometer (Thermo Fisher Scientific, Inc.). Subsequently, isolated RNA was reverse transcribed into cDNA using a PrimeScript RT reagent kit (Takara Bio, Inc.) and oligo primers (Takara Bio, Inc.). The conditions of RT were as follows: $38^{\circ} \mathrm{C}$ for $15 \mathrm{~min}$ and $85^{\circ} \mathrm{C}$ for $5 \mathrm{sec}$. Subsequently, qPCR was performed using the Power SYBR Green PCR Master mix (Takara Bio, Inc.). During qPCR, U6 RNA was used as the internal control to normalize the mRNA expression levels of HN1, ZEB2 and miR-132. The primer sequences were as follows: HN1 forward, 5'-CGCAGG CCCTAAACTACCAG-3' and reverse, 5'-TGCTACTGTCGA TGTGGACC-3'; ZEB2 forward, 5'-GCAGTGAGCATCGAA GAGTACC-3' and reverse, 5'-GGCAAAAGCATCTGGAGT TCCAG-3'; miR-132 forward, 5'-ACCGTGGCTTTCGATTG TT-3' and reverse, 5'-GAACATGTCTGCGTATCTC-3'; and U6 forward, 5'-GTGCTCGCTTCGGCAGCA-3' and reverse, 5'-CAAATATGGAACGCTTC-3'. The conditions of qPCR were as follows: Pre-denaturation at $95^{\circ} \mathrm{C}$ for $30 \mathrm{sec}, 35$ cycles of denaturation at $94^{\circ} \mathrm{C}$ for $15 \mathrm{sec}$, annealing at $56^{\circ} \mathrm{C}$ for $45 \mathrm{sec}$ and extension at $72^{\circ} \mathrm{C}$ for $45 \mathrm{sec}$. At the end of each qPCR cycle, the cycle threshold $(\mathrm{Cq})$ value was acquired. The relative mRNA expression of HN1, ZEB2 and miR-132 was calculated using the $2^{-\Delta \Delta \mathrm{Cq}}$ method (35). Three independent experiments were performed.

Cell culture and transfection. A549 and H460 cells were purchased from American Type Culture Collection and incubated at $37^{\circ} \mathrm{C}$ with $5 \% \mathrm{CO}_{2}$. The culture medium was Dulbecco's modified Eagle's medium (DMEM; Gibco; Thermo Fisher Scientific, Inc.), supplemented with $10 \%$ foetal bovine serum (FBS; Gibco; Thermo Fisher Scientific, Inc.).

For hesperidin treatment experiments, the cells were seeded into $96-$ well plates at a density of $1 \times 10^{5}$ cells/well and incubated at $37^{\circ} \mathrm{C}$ and $5 \% \mathrm{CO}_{2}$ for $12 \mathrm{~h}$, followed by treatment with 1 or $2.5 \mu \mathrm{M}$ hesperidin for $48 \mathrm{~h}$ at room temperature. The cells were harvested after $48 \mathrm{~h}$ of treatment and analysed. For transfection experiments, the cells were seeded into 6-well plates at a density of $1 \times 10^{4}$ cells/well and incubated at $37^{\circ} \mathrm{C}$ and $5 \% \mathrm{CO}_{2}$ overnight until the cells were $70-80 \%$ confluent. On the following day, the cells were transfected with miR-132 mimic, HN1 small interfering RNA (siRNA), ZEB2 siRNA, miRNA negative controls (NCs) or siRNA control (all from 

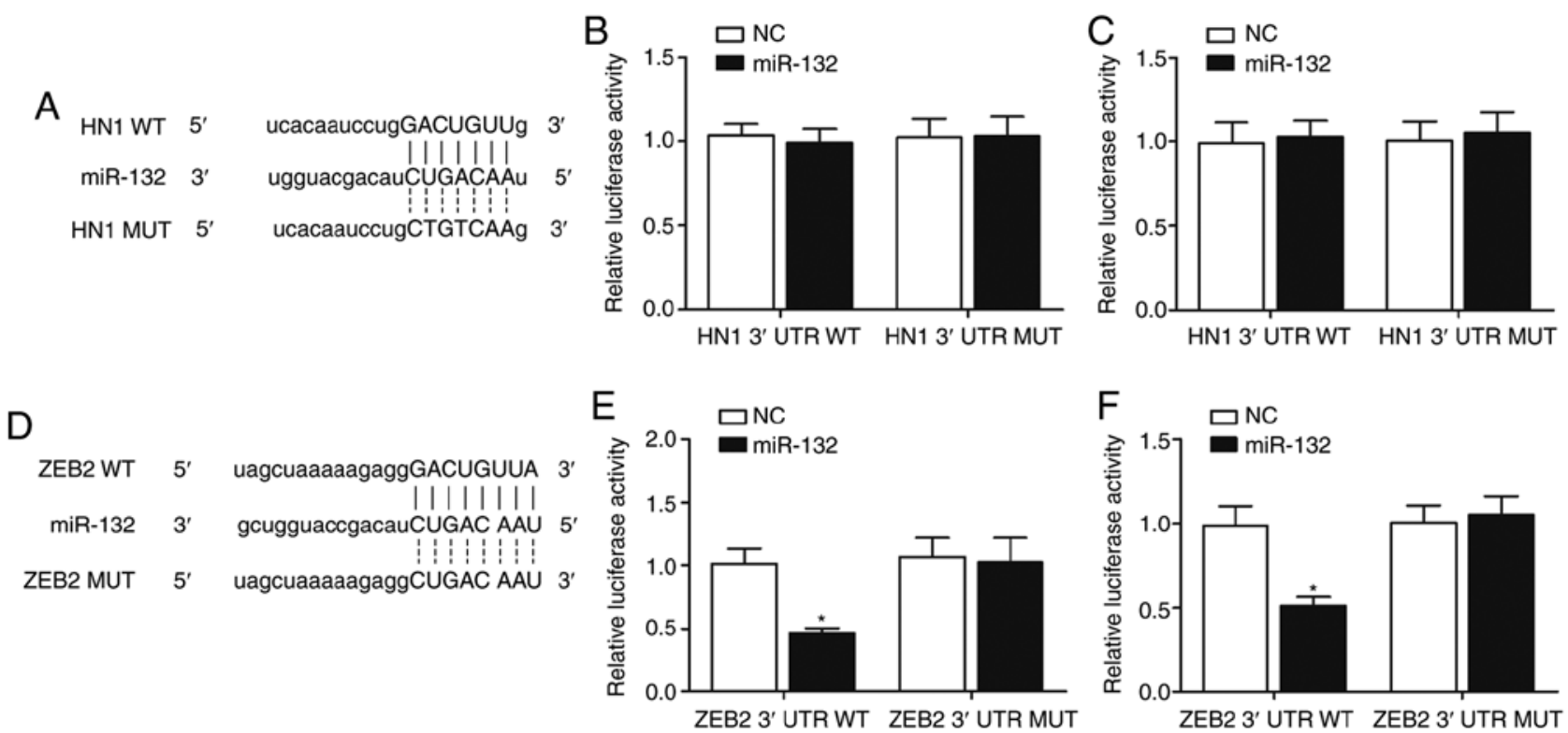

Figure 1. ZEB2 is a target gene of miR-132. (A) HN1 was predicted as a target gene of miR-132 with a potential miR-132 binding site located in the HN1 3'-UTR. (B) A549 and (C) H460 cells transfected with miR-132 mimic and the WT or MUT 3'-UTR of HN1 exhibited no change in their luciferase activity. (D) ZEB2 was predicted as a target gene of miR-132 with a potential miR-132 binding site located in the ZEB2 3'-UTR. miR-132 mimic decreased the luciferase activity of the WT but not the MUT ZEB2 3'-UTR in (E) A549 and (F) H460 cells. * P<0.05 vs. NC group. ZEB2, zinc finger E-box binding homeobox 2; miR-132, microRNA-132; HN1, neurological expressed 1; 3'-UTR, 3'-untranslated region; WT, wild-type; MUT, mutant; NC, negative control.

Santa Cruz Biotechnology, Inc.) using Lipofectamine 2000 (Invitrogen; Thermo Fisher Scientific, Inc.) according to the manufacturer's instructions. After $48 \mathrm{~h}$ of transfection, the cells were collected to analyse the expression of target genes. All experiments were performed in triplicate.

Cytotoxicity assay. Transfected A549 and H460 cells were seeded into 48-well plates and cultured in DMEM overnight. Subsequently, 5 mg/ml MTT (Sigma-Aldrich; Merck KGaA) was added to each well, and the incubation was continued for a further 4 h. Subsequently, DMSO (Sigma-Aldrich; Merck $\mathrm{KGaA}$ ) was added to each well, and the plates were placed on a shaker for $10 \mathrm{~min}$. Finally, the optical density in each well was measured using a spectrophotometer at a wavelength of $490 \mathrm{~nm}$. All experiments were run in triplicate.

Colony formation assay. After $48 \mathrm{~h}$ of transfection, the cells were detached with $0.25 \%$ trypsin and resuspended in 2X DMEM (containing 20\% FBS, Gibco; Thermo Fisher Scientific, Inc.) to adjust the cell concentration to $4 \times 10^{4}$ cells $/ \mathrm{ml}$. Hard agar medium (lower layer) and soft agar medium (upper layer) were then prepared by mixing a $1.0 \%$ soft agar solution and a $0.7 \%$ soft agar solution with the $2 \mathrm{X}$ DMEM medium in a 1:1 ratio, respectively. Subsequently, the hard agar medium was added into a six-well plate $(1.5 \mathrm{ml} /$ well $)$ and cooled to room temperature, followed by the addition of the soft agar medium on top of the basal medium to form a double agar layer. Following the solidification of the upper agar layer, the plate was incubated at $37^{\circ} \mathrm{C}$ with $5 \% \mathrm{CO}_{2}$ for $14-21$ days, with medium added at regular intervals to maintain the moisture on the agar surface. The formation of the cell colonies was observed under an inverted microscope (magnification x200). The stained colonies were photographed, and the number of clones was counted and analysed using Image-Pro Plus 6.0 software (Media Cybernetics, Inc.). All experiments were run in triplicate.

Vector construction, mutagenesis and luciferase assay. A combination of bioinformatics tools, including TargetScan (www.targetscan.org) and the miRNA database miRBase (www.mirbase.org), were used to identify the potential regulatory relationship between miR-132 and HN1, as well as the regulatory relationship between miR-132 and ZEB2. After PCR was carried out to amplify the 3'-UTR of HN1 and ZEB2 containing the binding sites of miR-132, the PCR products were inserted into pcDNA vectors (Promega Corporation) using a TA cloning kit (Invitrogen; Thermo Fisher Scientific, Inc.) to generate the expression constructs of wild-type HN1 and ZEB2. In addition, the Quick Change XL site-directed mutagenesis kit (Stratagene; Merck KGaA) was used to mutate the miR-132 binding sites in the 3'-UTR of HN1 and ZEB2. The mutated sequences were also amplified by PCR and inserted into pcDNA vectors to generate the expression constructs of mutant HN1 and ZEB2. Subsequently, A549 and H460 cells were co-transfected with miR-132 and wild-type/mutant ZEB2 or miR-132 and wild-type/mutant HN1 using Lipofectamine 2000, following the manufacturer's instructions. At $48 \mathrm{~h}$ post-transfection, the luciferase activity was measured using a Dual-Luciferase Reporter assay system (Promega Corporation) on a luminometer. The activity of Renilla luciferase was used as an internal control. Each experiment was repeated three times.

Western blot analysis. Following two washes with PBS, NSCLC tissues and transfected cells were ground in liquid nitrogen and homogenized with the addition of lysis buffer (BioSharp, Inc.). Subsequently, sample tissues were centrifuged at $241.49 \mathrm{x} \mathrm{g}$ for $30 \mathrm{~min}$ at $4^{\circ} \mathrm{C}$ to remove debris. The 
A

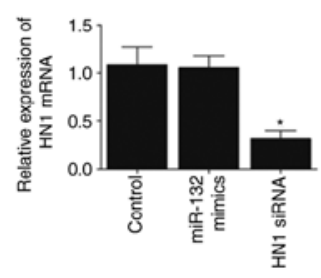

F

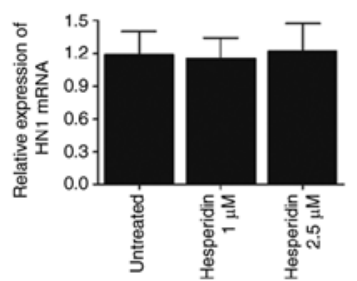

K

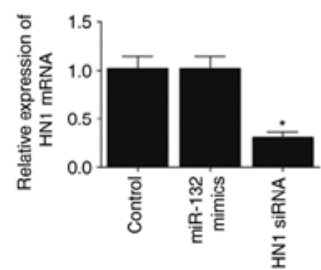

P

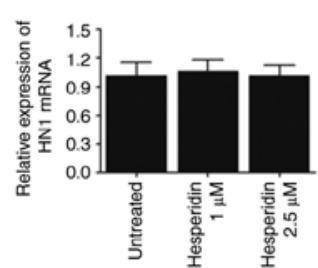

B

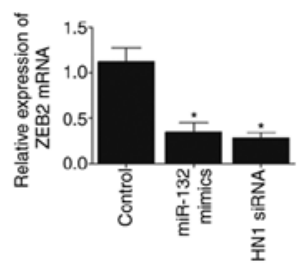

G

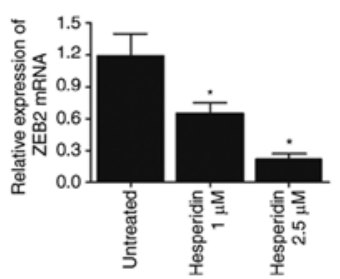

L

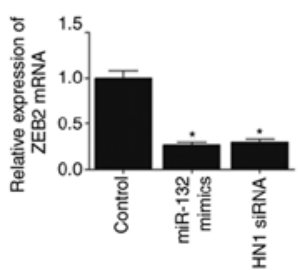

Q

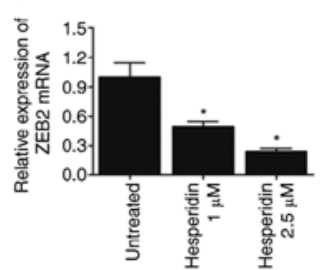

C

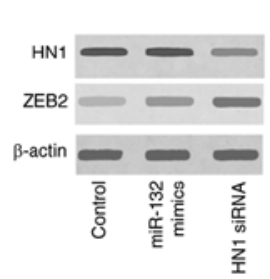

$\mathrm{H}$

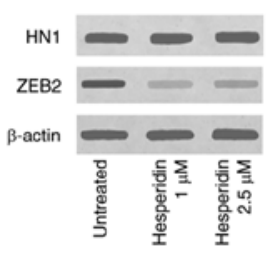

D

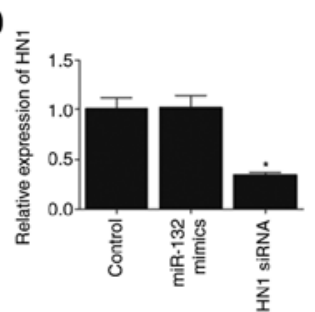

E

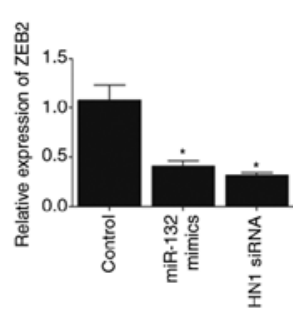

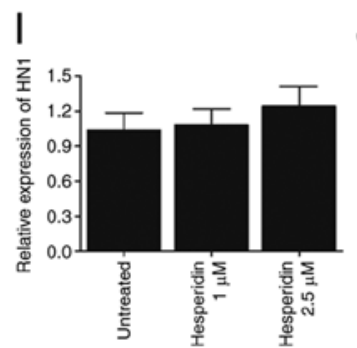

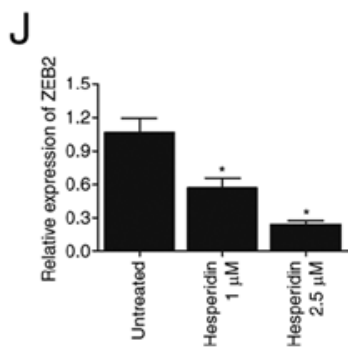

M

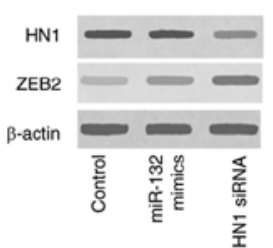

$\mathrm{N}$

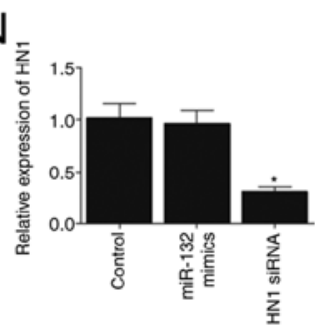

R

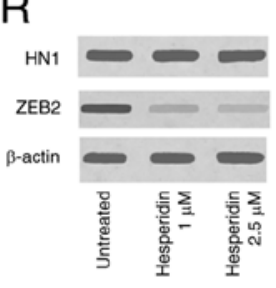

$S$

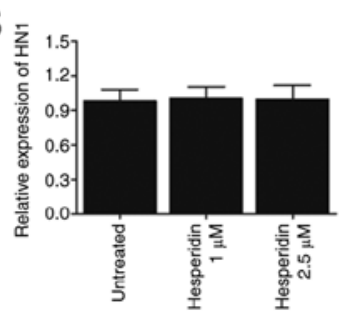

O

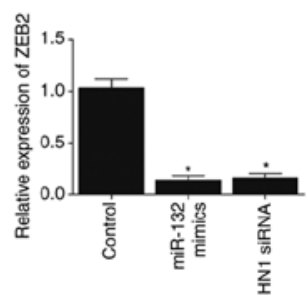

$\mathrm{T}_{\text {\% }}$

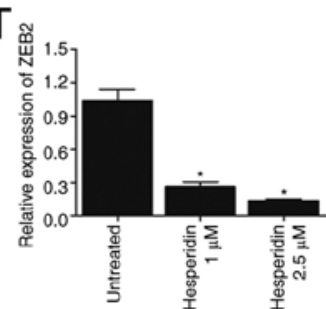

Figure 2. Effect of miR-132 and hesperidin on ZEB2 expression in A549 and H460 cells. (A) In A549 cells, miR-132 mimic demonstrated no effect on the mRNA expression of HN1, whereas HN1 siRNA reduced the mRNA expression of HN1. (B) In A549 cells, miR-132 mimic and ZEB2 siRNA both decreased the mRNA expression of ZEB2. (C and D) In A549 cells, miR-132 mimic demonstrated no effect on the protein expression of HN1, but reduced the protein expression of ZEB2. (E) In A549 cells, miR-132 mimic and ZEB2 siRNA both decreased the protein expression of ZEB2. (F) Treatment with hesperidin exhibited no effect on the mRNA expression of HN1 in A549 cells. (G) Treatment with hesperidin decreased the mRNA expression of ZEB2 in a concentration-dependent manner in A549 cells. (H) Western blot results of HN1 and ZEB2 protein in A549 cells treated with different concentrations of hesperidin. (I) Treatment with hesperidin demonstrated no effect on the protein expression of HN1 in A549 cells. (J) Treatment with hesperidin decreased the protein expression of ZEB2 in a concentration-dependent manner in A549 cells. (K) In H460 cells, miR-132 mimic had no effect on the mRNA expression of HN1, whereas HN1 siRNA reduced the mRNA expression of HN1. (L) In H460 cells, miR-132 mimic and ZEB2 siRNA both decreased the mRNA expression of ZEB2. (M and N) In H460 cells, miR-132 mimic exhibited no effect on the protein expression of HN1, but HN1 siRNA reduced the expression. (O) In H460 cells, miR-132 mimic and ZEB2 siRNA both decreased the protein expression of ZEB2. (P) Treatment with hesperidin demonstrated no effect on the mRNA expression of HN1 in H460 cells. (Q) Treatment with hesperidin decreased the mRNA expression of ZEB2 in a concentration-dependent manner in H460 cells. (R) Western blot results of HN1 and ZEB2 protein in H460 cells treated with different concentrations of hesperidin. (S) Treatment with hesperidin had no effect on the protein expression of HN1, (T) but decreased the protein expression of ZEB2 in a concentration-dependent manner in H460 cells. " $\mathrm{P}<0.05$ vs. control/untreated group. ZEB2, zinc finger E-box binding homeobox 2; miR-132, microRNA-132; HN1, neurological expressed 1; siRNA, small interfering RNA.

supernatant was collected to measure the total protein concentration using a bicinchoninic acid kit (BioTeke Corporation), according to the manufacturer's protocol. Protein $(50 \mu \mathrm{g})$ from each sample was then mixed with $2 \mathrm{X}$ SDS loading buffer and boiled at $100^{\circ} \mathrm{C}$ for $5 \mathrm{~min}$. following separation by $10 \%$ SDS-PAGE, the protein was transferred onto a polyvinylidene fluoride membrane. The membrane was then blocked with $5 \%$ skimmed milk for $1 \mathrm{~h}$ at room temperature and incubated overnight at $4^{\circ} \mathrm{C}$ with diluted (1:100) primary antibodies against HN1 (cat. no. ab126705; Abcam), ZEB2 (cat. no. ab223688; Abcam) and GAPDH (cat. no. ab8245;
Abcam). Subsequently, the membrane was washed three times with TBST and incubated for $1 \mathrm{~h}$ at room temperature with rabbit anti-mouse horseradish peroxidase-labelled secondary antibodies (1:2,000; cat. no. ab6728; Abcam). The membrane was then developed using an enhanced chemiluminescence reagent (Biomiga, Inc.) and visualized using an X-ray apparatus. GAPDH served as the internal control to quantify the relative protein expression levels of $\mathrm{HN} 1$ and ZEB2. ImageJ software (version 1.41; National Institutes of Health) was used to quantify the western blots. Each experiment was repeated three times. 

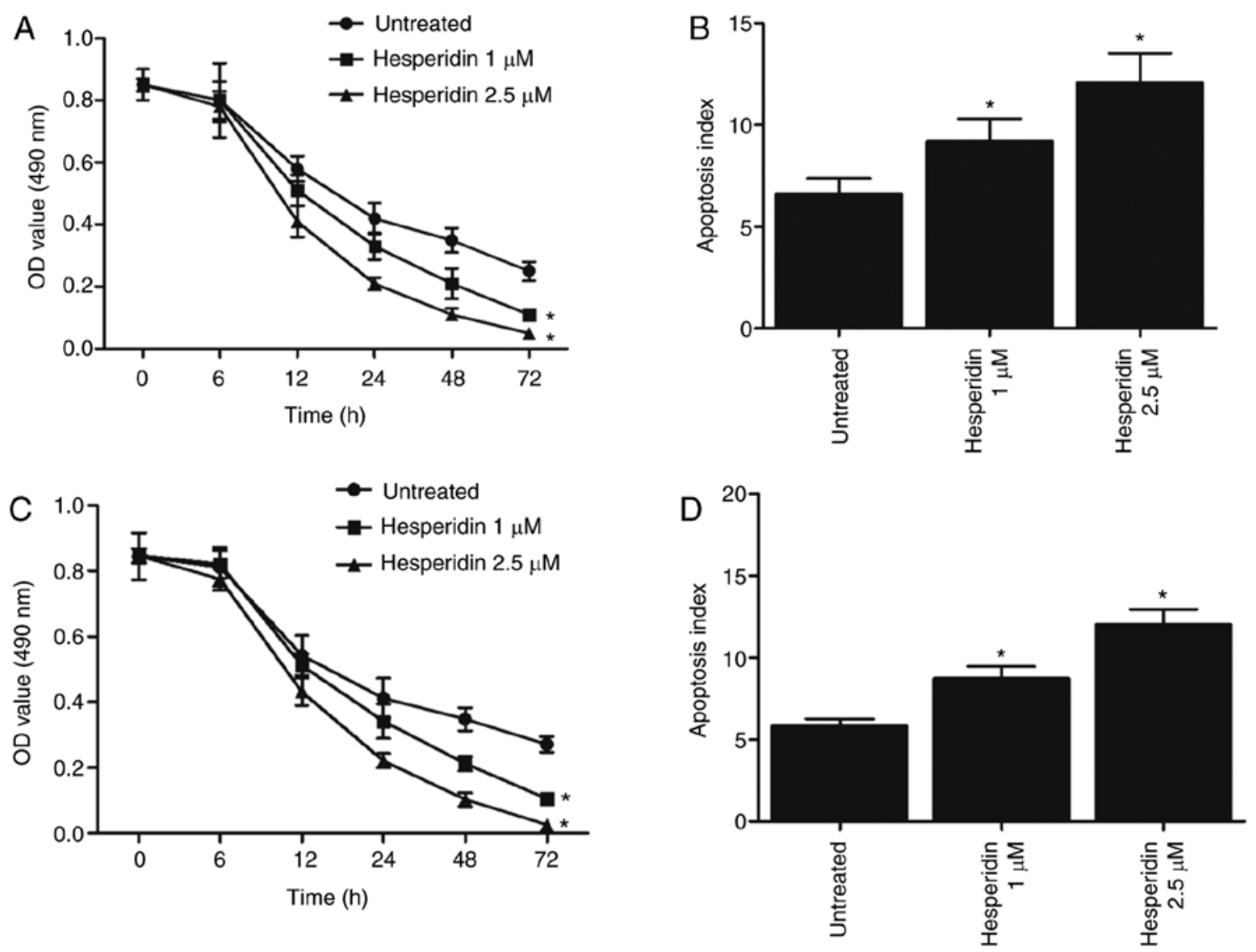

Figure 3. Treatment with hesperidin affects the proliferation and apoptosis of A549 and H460 cells. (A) Hesperidin inhibited the proliferation of A549 cells in a dose-dependent manner. (B) Hesperidin promoted the apoptosis of A549 cells in a dose-dependent manner. (C) Hesperidin inhibited the proliferation of $\mathrm{H} 460$ cells in a dose-dependent manner. (D) Hesperidin promoted the apoptosis of $\mathrm{H} 460$ cells in a dose-dependent manner. "P $<0.05$ vs. untreated group. OD, optical density.

Apoptosis analysis by flow cytometry and TUNEL staining. FITC Annexin V Apoptosis Detection kit I (cat. no. 556547; BD Biosciences) was used according to the manufacture's protocol to analyse the apoptosis of transfected A549 and H460 cells, with a flow cytometer (Thermo Fisher Scientific, Inc.). In addition, apoptosis was assessed using a TUNEL Alexa Fluor ${ }^{\mathrm{TM}} 488$ Imaging Assay kit (cat. no. C10245; Invitrogen; Thermo Fisher Scientific, Inc.), according to the manufacturer's protocol. Each experiment was repeated three times.

Statistical analysis. All data are presented as the mean \pm standard error of the mean. One-way ANOVA and an unpaired Student's t-test were utilized to compare results among multiple groups and between two groups, respectively. All statistical analysis was performed using SPSS version 19.0 (IBM Corp.). $\mathrm{P}<0.05$ was considered to indicate a statistically significant difference.

\section{Results}

ZEB2 is a candidate target gene of miR-132. By performing computational analysis using TargetScan and the miRNA database miRBase, HN1 and ZEB2 were identified as possible target genes of miR-132, with both 3'-UTRs of HN1 (Fig. 1A) and ZEB2 (Fig. 1D) containing a potential binding site for miR-132. To further investigate whether miR-132 targets HN1 and ZEB2 directly, luciferase reporter vectors containing wild-type or mutant 3'-UTRs of HN1 or ZEB2 were created and co-transfected into A549 and H460 cells with miR-132 mimic or NC. The luciferase activity of wild-type or mutant HN1 3'-UTR was not significantly different in A549 (Fig. 1B) and H460 (Fig. 1C) cells co-transfected with miR-132 mimic compared with the NC. By contrast, co-transfection of A549 (Fig. 1E) and H460 (Fig. 1F) cells with miR-132 mimic and wild-type ZEB2 3'-UTR significantly reduced the luciferase activity of the cells, suggesting that ZEB2 is a target gene of miR-132.

Effect of miR-132 and hesperidin on ZEB2 expression. To further investigate the effect of miR-132 on ZEB2 and HN1 expression, the expression levels of ZEB2 and HN1 were measured in A549 cells (Fig. 2A-J) and H460 cells (Fig. 2K-T) transfected with miR-132 orZEB2/HN1 siRNA. The successful transfections of miR-132 mimic (Fig. S1A and D), HN1 siRNA (Fig. S1B and E) and ZEB2 siRNA (Fig. S1C and F) in A549 and H460 cells were validated by RT-qPCR. In addition, the cells were treated with different concentrations of hesperidin before measuring the expression of ZEB2 and HN1. As presented in Fig. 2, miR-132 mimic had no effect on the mRNA (Fig. 2A and K) and protein (Fig. 2C, D, M and N) expression levels of HN1, while transfection with HN1 siRNA significantly decreased the expression of HN1 mRNA (Fig. 2A and K) and protein (Fig. 2C, D, M and N) in A549 

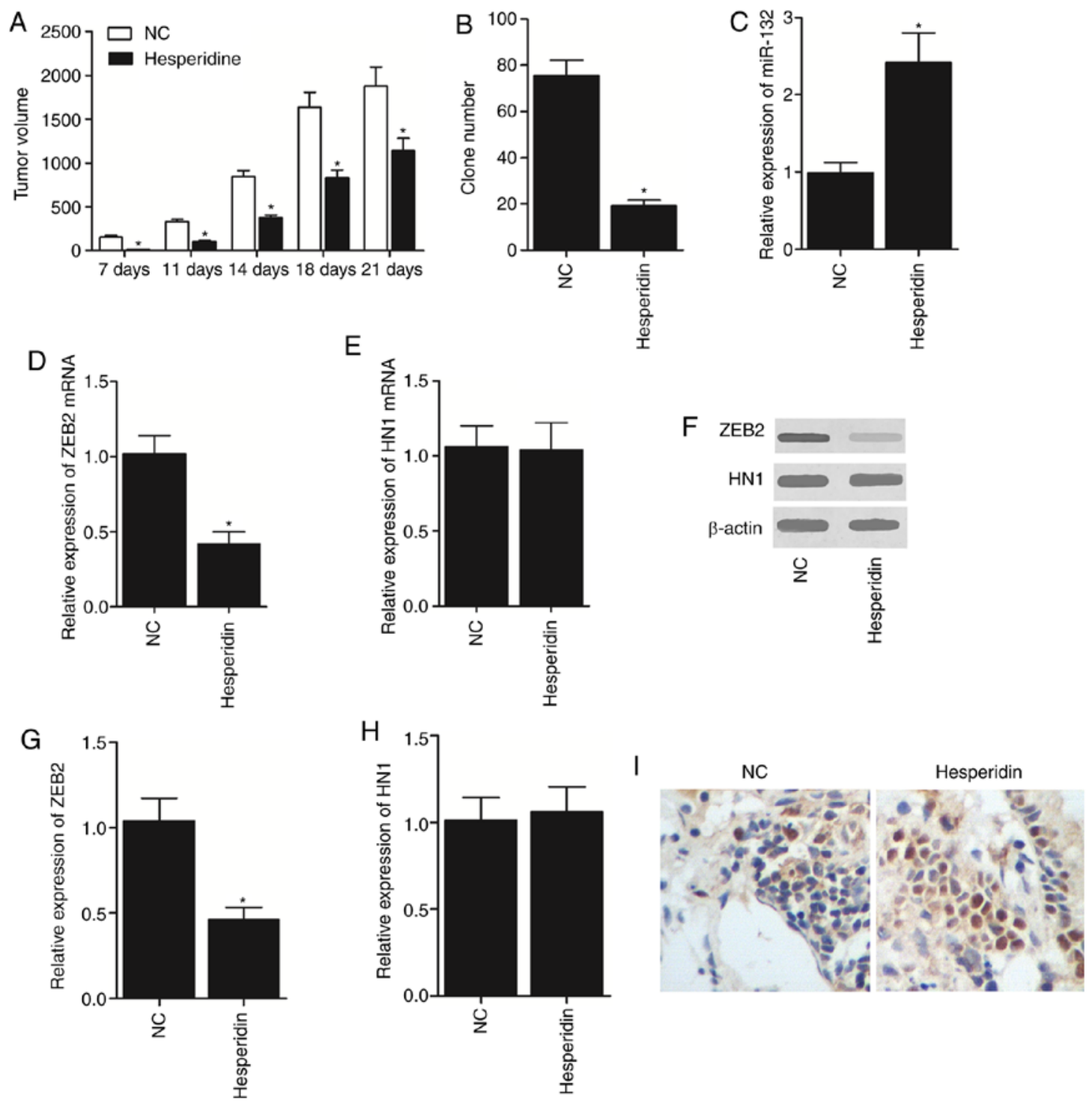

Figure 4. Effect of hesperidin on the tumor volume and colony formation efficiency of NSCLC, and on HN1 and ZEB2 expression in rats transplanted with NSCLC cells. (A) The tumor volume in rats transplanted with NSCLC cells and treated with hesperidin was smaller compared with that in rats transplanted with NSCLC cells alone. (B) Treatment with hesperidin significantly reduced the colony formation efficiency of NSCLC cells. (C) Hesperidin increased miR-132 expression in the rats. (D) Hesperidin decreased the mRNA expression of ZEB2 in rats transplanted with NSCLC cells. (E) Hesperidin demonstrated no effect on the mRNA expression of HN1 in rats transplanted with NSCLC cells. (F) Hesperidin decreased the protein expression of ZEB2 in rats transplanted with NSCLC cells, but showed no effect on the protein expression of HN1. (G) Quantification of the western blots demonstrated that hesperidin significantly decreased the protein expression of ZEB2 in rats transplanted with NSCLC cells. (H) Quantification of the western blots demonstrated hesperidin had no effect on the protein expression of HN1 in rats transplanted with NSCLC cells. (I) Hesperidin increased the apoptosis of NSCLC cells (magnification x200). "P<0.05 vs. NC group. NSCLC, non-small cell lung cancer; ZEB2, zinc finger E-box binding homeobox 2; miR-132, microRNA-132; HN1, neurological expressed 1; $\mathrm{NC}$, negative control.

and $\mathrm{H} 460$ cells. By contrast, the transfection of A549 and H460 cells with miR-132 mimic or ZEB2 siRNA significantly reduced the expression of ZEB2 mRNA (Fig. 2B and L) and protein (Fig. 2C, E, M and O). In addition, treatment of cells with various doses of hesperidin demonstrated no effect on the expression of HN1 mRNA (Fig. 2F and P) and protein (Fig. 2H, I, R and S), while hesperidin treatment significantly reduced the expression of ZEB2 mRNA (Fig. 2G and Q) and protein (Fig. $2 \mathrm{H}, \mathrm{J}, \mathrm{R}$ and $\mathrm{T}$ ) in a concentration-dependent manner. In summary, these results indicate that miR-132 and hesperidin inhibit ZEB2 expression.

Effect of hesperidin on cell proliferation and apoptosis. MTT assay and flow cytometry analysis were used to evaluate the effect of hesperidin ( 1 and $2.5 \mu \mathrm{M})$ on the viability and apoptosis of A549 and H460 cells. The results demonstrated that treatment with hesperidin significantly inhibited the proliferation of A549 (Fig. 3A) and H460 (Fig. 3C) cells, and significantly promoted apoptosis (Fig. 3B and D) in a dose-dependent manner.

Effect of hesperidin on NSCLC. To investigate the effect of hesperidin on NSCLC, experimental rats were randomly assigned to two groups. In one group, the rats were transplanted with NSCLC cells. In another group, the rats were transplanted with NSCLC cells and treated with hesperidin at the same time. As presented in Fig. 4, the rats transplanted with NSCLC cells and treated with hesperidin had 
significantly smaller tumour volumes compared with the rats that only received NSCLC cell transplantation (Fig. 4A). In addition, treatment with hesperidin significantly decreased the colony formation efficiency of NSCLC cells (Fig. 4B), and the TUNEL staining also revealed more apoptotic NSCLC cells upon the administration of hesperidin compared with the untreated NSCLC cells (Fig. 4I). Furthermore, RT-qPCR, western blotting and IHC were used to detect the levels of miR-132, HN1 and ZEB2 in the rats from the two groups. The results demonstrated that hesperidin treatment significantly increased miR-132 expression (Fig. 4C), and significantly decreased the ZEB2 mRNA (Fig. 4D) and protein (Fig. 4F and G) expression levels in rats transplanted with NSCLC cells. Furthermore, it was identified that the mRNA (Fig. 4E) and protein (Fig. 4F and H) expression levels of HN1 were similar in the two groups of rats.

\section{Discussion}

Hesperidin is a member of the flavanone family, and flavanones are mainly produced in citrus fruits. Due to its effects on disease treatment and prevention, hesperidin has become a topic of interest in recent years (36). In particular, the anticancer properties of hesperidin have been studied extensively (36). Hesperidin also exerts other biological effects, including anti-inflammatory, antioxidant and anti-mutagenic effects $(37,38)$. In addition, hesperidin can inhibit the proliferation of cancer cells in oral cancer (39). Furthermore, hesperidin can suppress cancer cell invasion by inhibiting the expression of proteins participating in EMT and members of the matrix metallopeptidase (MMP) protein family $(13,40,41)$. A previous study demonstrated that hesperidin induces the apoptosis of A549 cells by activating the proteins that participate in mitochondrial apoptosis and by regulating the expression of molecules associated with cell cycle progression (15). Another study indicated that SDF-1/CXCR-4 signalling can block the role of hesperidin in suppressing the invasion and migration of A549 cells (14). Based on these results, hesperidin may be a promising agent in the treatment of NSCLC. Balakrishnan and Menon (40) demonstrated that hesperidin induces the apoptosis of colon cancer cells by increasing the activity of caspase- 9 and caspase-3. Furthermore, it has been reported that hesperidin induces the apoptosis of HepG2 cells by activating ERK1/2 signalling (42). The present study investigated the effect of hesperidin on cell proliferation and apoptosis using an MTT assay and flow cytometry analysis. The results demonstrated that hesperidin treatment repressed cell proliferation and promoted cell apoptosis in a dose-dependent manner. Furthermore, RT-qPCR and western blot analysis were performed to detect the effect of miR-132 and hesperidin on the expression of HN1 and ZEB2. The results indicated that both miR-132 and hesperidin could significantly decrease the expression of ZEB2, while showing no effect on HN1 expression.

It has previously been reported that hesperidin can suppress the growth and induce the apoptosis of cancer cells (14). In particular, hesperidin triggers apoptotic signalling in NCI-H358 and A549 cells $(14,43)$. In addition, hesperidin exerts minimal cytotoxicity in MRC-5 cells, a type of normal lung fibroblast (43). Hesperidin has been shown to increase the expression of caspase- 3 enzyme and reduce the level of MMP, which is closely associated apoptosis (44). The results of FITC-Annexin V/PI staining in a recent study also demonstrated the pro-apoptotic effect of hesperidin in NSCLC cells (15).

miRNA-132 has been reported to regulate the in vivo expression of proteins associated with inflammation. A previous study demonstrated that miRNA-132 enhances the signalling of the cholinergic anti-inflammatory pathway (45). It was demonstrated that miRNA-132 antagonists not only reduced the efficacy of hesperidin in the treatment of depression but also blocked its anti-inflammatory effects. According to the data published in previous studies, it was hypothesized that hesperidin can restore the negative feedback between pro-inflammatory cytokines and miRNA-132 (46,47). The present study transplanted NSCLC cells into rats and treated some rats with hesperidin at the same time to investigate the effect of hesperidin on NSCLC. The results demonstrated that treatment with hesperidin decreased the tumour volume and colony formation efficiency of NSCLC cells by increasing miR-132 expression and decreasing ZEB2 expression. Furthermore, among multiple targets for miR-132 in cancer, ZEB2, as an inhibitor of E-cadherin transcription, was reported to play an essential role in EMT and acts as a direct target of miR-132, and the ectopic expression of miR-132 significantly reduced the invasiveness of CRC cells and inhibited their EMT process (21).

It is worth noting that there were limitations of the present study. Due to the fact that an overdose of hesperidin may kill animals, while a low dose of hesperidin may exhibit no significant therapeutic effect on the animals, a preliminary test was performed to determine the dose to treat the animals (data not shown). However, only two animals in each group were used to determine the dose in the preliminary test, which was an inappropriate sample size for statistical analysis. Furthermore, the present findings were not validated with human subjects. It would be necessary to recruit human subjects and validate the administration of hesperidin in future studies.

In conclusion, the present study demonstrated that the administration of hesperidin upregulates the expression of miR-132, a potential regulator of HN1, TGF- $\beta 1$ and ZEB2. Since HN1, TGF- $\beta 1$ and ZEB2 have been reported to be involved in the pathogenesis of NSCLC, the administration of hesperidin may alleviate NSCLC by inhibiting the proliferation and promoting the apoptosis of NSCLC cells via the miR-132/ZEB2 pathway.

\section{Acknowledgements}

Not applicable.

\section{Funding}

No funding was received.

\section{Availability of data and materials}

The datasets used and/or analysed during the present study are available from the corresponding author on reasonable request. 


\section{Authors' contributions}

ST and LD designed the study. AH supervised the study. PT and WL collected the references. ST, LD, YM and PT collected the experimental data, AH, WL and YM collected the patient data. AH, JW, WL and XH analysed the data. ST, YM and JW wrote the manuscript. AH improved the manuscript. All authors read and approved the final manuscript.

\section{Ethics approval and consent to participate}

All animal experiments in the present study were approved by the Animal Ethics Committee of Yantai Hospital of Traditional Chinese Medicine (approval no. YTYX16X316).

\section{Patient consent for publication}

Not applicable.

\section{Competing interests}

The authors declare that they have no competing interests.

\section{References}

1. DeSantis CE, Lin CC, Mariotto AB, Siegel RL, Stein KD, Kramer JL, Alteri R, Robbins AS and Jemal A: Cancer treatment and survivorship statistics, 2014. CA Cancer J Clin 64: 252-271, 2014.

2. He J, Shen J, Yang C, Jiang L, Liang W, Shi X, Xu X and He J: Adjuvant chemotherapy for the completely resected stage IB nonsmall cell lung cancer: A Systematic review and meta-analysis. Medicine (Baltimore) 94: e903, 2015.

3. Lai WY, Wang WY, Chang YC, Chang CJ, Yang PC and Peck K: Synergistic inhibition of lung cancer cell invasion, tumor growth and angiogenesis using aptamer-siRNA chimeras. Biomaterials 35: 2905-2914, 2014.

4. Hong S, Tan M, Wang S, Luo S, Chen Y and Zhang L: Efficacy and safety of angiogenesis inhibitors in advanced non-small cell lung cancer: A systematic review and meta-analysis. J Cancer Res Clin Oncol 141: 909-921, 2015.

5. Qin Y, Zhang Q, Lee S, Zhong WL, Liu YR, Liu HJ, Zhao D, Chen S, Xiao T, Meng J, et al: Doxycycline reverses epithelial-to-mesenchymal transition and suppresses the proliferation and metastasis of lung cancer cells. Oncotarget 6: 40667-40679, 2015.

6. Fischer KR, Durrans A, Lee S, Sheng J, Li F, Wong ST, Choi H, El Rayes T, Ryu S, Troeger J, et al: Epithelial-to-mesenchymal transition is not required for lung metastasis but contributes to chemoresistance. Nature 527: 472-476, 2015.

7. Li Y, Chao Y, Fang Y, Wang J, Wang M, Zhang H, Ying M, Zhu X and Wang H: MTA1 promotes the invasion and migration of non-small cell lung cancer cells by downregulating miR-125b. J Exp Clin Cancer Res 32: 33, 2013.

8. Chen L, Gibbons DL, Goswami S, Cortez MA, Ahn YH, Byers LA, Zhang X, Yi X, Dwyer D, Lin W, et al: Metastasis is regulated via microRNA-200/ZEB1 axis control of tumour cell PD-L1 expression and intratumoral immunosuppression. Nat Commun 5: 5241, 2014.

9. Li H, Chen Y, Xu N, Yu M, Tu X, Chen Z, Lin M, Xie B, Fu J and Han L: AMD3100 inhibits brain-specific metastasis in lung cancer via suppressing the SDF-1/CXCR4 axis and protecting blood-brain barrier. Am J Transl Res 9: 5259-5274, 2017.

10. Xie S, Zeng W, Fan G, Huang J, Kang G, Geng Q, Cheng B, Wang W and Dong P: Effect of CXCL12/CXCR4 on increasing the metastatic potential of non-small cell lung cancer in vitro is inhibited through the downregulation of CXCR4 chemokine receptor expression. Oncol Lett 7: 941-947, 2014

11. Kamaraj S, Ramakrishnan G, Anandakumar P, Jagan S and Devaki T: Antioxidant and anticancer efficacy of hesperidin in benzo(a)pyrene induced lung carcinogenesis in mice. Invest New Drugs 27: 214-22, 2009.
12. Khamis AAA, Ali EMM,El-Moneim MAA, Abd-Alhaseeb MM, El-Magd MA and Salim EI: Hesperidin, piperine and bee venom synergistically potentiate the anticancer effect of tamoxifen against breast cancer cells. Biomed Pharmacother 105: 1335-1343, 2018

13. Roohbakhsh A, Parhiz H, Soltani F, Rezaee R and Iranshahi M: Molecular mechanisms behind the biological effects of hesperidin and hesperetin for the prevention of cancer and cardiovascular diseases. Life Sci 124: 64-74, 2015

14. Xia R, Xu G, Huang Y, Sheng X, Xu X and Lu H: Hesperidin suppresses the migration and invasion of non-small cell lung cancer cells by inhibiting the SDF-1/CXCR-4 pathway. Life Sci 201: 111-120, 2018.

15. Xia R, Sheng $\mathrm{X}, \mathrm{Xu} \mathrm{X}, \mathrm{Yu} \mathrm{C}$ and $\mathrm{Lu} \mathrm{H}$ : Hesperidin induces apoptosis and G0/G1 arrest in human non-small cell lung cancer A549 cells. Int J Mol Med 1: 464-472, 2017.

16. Kataoka M and Wang DZ: Non-coding RNAs including miRNAs and lncRNAs in cardiovascular biology and disease. Cells 3: 883-898, 2014

17. Moreno-Moya JM, Vilella F and Simon C: MicroRNA: Key gene expression regulators. Fertil Steril 101: 1516-1523, 2014.

18. Ell B and Kang Y: MicroRNAs as regulators of bone homeostasis and bone metastasis. Bonekey Rep 3: 549, 2014.

19. Janaki Ramaiah M, Lavanya A, Honarpisheh M, Zarea M, Bhadra U and Bhadra MP: MiR-15/16 complex targets p70S6 kinase 1 and controls cell proliferation in MDA-MB-231 breast cancer cells. Gene 552: 255-264, 2014.

20. Li W, Zang W, Liu P, Wang Y, Du Y, Chen X, Deng M, Sun W, Wang L, Zhao G and Zhai B: MicroRNA-124 inhibits cellular proliferation and invasion by targeting Ets-1 in breast cancer. Tumour Biol 35: 10897-10904, 2014.

21. You J, Li Y, Fang N, Liu B, Zu L, Chang R, Li X and Zhou Q: MiR-132 suppresses the migration and invasion of lung cancer cells via targeting the EMT regulator ZEB2. PLoS One 9: e91827, 2014

22. Hansen KF, Karelina K, Sakamoto K, Wayman GA, Impey S and Obrietan K: miRNA-132: A dynamic regulator of cognitive capacity. Brain Struct Funct 218: 817-831, 2013.

23. Marler KJ, Suetterlin P, Dopplapudi A, Rubikaite A, Adnan J, Maiorano NA, Lowe AS, Thompson ID, Pathania M, Bordey A, et al: BDNF promotes axon branching of retinal ganglion cells via miRNA-132 and p250GAP. J Neurosci 34: 969-979, 2014.

24. Marques-Rocha JL, Samblas M, Milagro FI, Bressan J, Martínez JA and Marti A: Noncoding RNAs, cytokines, and inflammation-related diseases. FASEB J 29: 3595-3611, 2015.

25. Li M, Shao H, Zhang X and Qin B: Hesperidin alleviates lipopolysaccharide-induced neuroinflammation in mice by promoting the miRNA-132 pathway. Inflammation 39: 1681-1689, 2016.

26. Yi LT, Li J, Liu BB, Luo L, Liu Q and Geng D: BDNF-ERK-CREB signalling mediates the role of miR-132 in the regulation of the effects of oleanolic acid in male mice. J Psychiatry Neurosci 39: 348-359, 2014.

27. Pan B and Liu Y: Effects of duloxetine on microRNA expression profile in frontal lobe and hippocampus in a mouse model of depression. Int J Clin Exp Pathol 8: 15454-15461, 2015.

28. You J, Li Y, Fang N, Liu B, Zu L, Chang R, Li X and Zhou Q: MiR-132 suppresses the migration and invasion of lung cancer cells via targeting the EMT regulator ZEB2. PLoS One 9: e91827, 2014

29. Zhang B, Lu L, Zhang X, Ye W, Wu J, Xi Q and Zhang X: Hsa-miR-132 regulates apoptosis in non-small cell lung cancer independent of acetylcholinesterase. J Mol Neurosci 53: 335-344, 2014.

30. Zhang JX, Zhai JF, Yang XT and Wang J: MicroRNA-132 inhibits migration, invasion and epithelial-mesenchymal transition by regulating TGF $31 / \mathrm{Smad} 2$ in human non-small cell lung cancer. Eur Rev Med Pharmacol Sci 20: 3793-3801, 2016.

31. Chen YK, Wang HC, Ho CT, Chen HY, Li S, Chan HL, Chung TW, Tan KT, Li YR and Lin CC: 5-demethylnobiletin promotes the formation of polymerized tubulin, leads to G2/M phase arrest and induces autophagy via JNK activation in human lung cancer cells. J Nutr Biochem 26: 484-504, 2015.

32. Jayaprakasha GK, Mandadi KK, Poulose SM, Jadegoud Y, Nagana Gowda GA and Patil BS: Novel triterpenoid from Citrus aurantium L. possesses chemopreventive properties against human colon cancer cells. Bioorg Med Chem 16: 5939-5951, 2008.

33. Lee DH, Park KI, Park HS, Kang SR, Nagappan A, Kim JA, Kim EH, Lee WS, Hah YS, Chung HJ, et al: Flavonoids isolated from Korea Citrus aurantium L. induce G2/M phase arrest and apoptosis in human gastric cancer AGS cells. Evid Based Complement Alternat Med 2012: 515901, 2012. 
34. Leclere L, Fransolet M, Cote F, Cambier P, Arnould T, Van Cutsem P and Michiels C: Heat-modified citrus pectin induces apoptosis-like cell death and autophagy in HepG2 and A549 cancer cells. PLoS One 10: e0115831, 2015.

35. Livak KJ and Schmittgen TD: Analysis of relative gene expression data using real-time quantitative PCR and the 2(-Delta Delta $\mathrm{C}(\mathrm{T}))$ method. Methods 25: 402-408, 2001.

36. Yang T, Wan Z, Liu Z, Li H, Wang H, Lu N, Chen Z, Mei X and Ren X: In situ mineralization of anticancer drug into calcium carbonate monodisperse nanospheres and their $\mathrm{pH}$-responsive release property. Mater Sci Eng C Mater Biol Appl 63: 384-392, 2016.

37. Galati EM, Monforte MT, Kirjavainen S, Forestieri AM, Trovato A and Tripodo MM: Biological effects of hesperidin, a citrus flavonoid. (Note I): Antiinflammatory and analgesic activity. Farmaco 40: 709-712, 1994

38. Huang MT, Wood AW, Newmark HL, Sayer JM, Yagi H, Jerina DM and Conney AH: Inhibition of the mutagenicity of bay-region diol-epoxides of polycyclic aromatic hydrocarbons by phenolic plant flavonoids. Carcinogenesis 4: 1631-1637, 1983

39. Tanaka T, Makita H, Ohnishi M, Mori H, Satoh K, Hara A, Sumida T, Fukutani K, Tanaka T and Ogawa H: Chemoprevention of 4-nitroquinoline 1-oxide-induced oral carcinogenesis in rats by flavonoids diosmin and hesperidin, each alone and in combination. Cancer Res 57: 246-252, 1997.

40. Balakrishnan A and Menon VP: Effect of hesperidin on matrix metalloproteinases and antioxidant status during nicotine-induced toxicity. Toxicology 238: 90-98, 2007.

41. Kamaraj S, Anandakumar P, Jagan S, Ramakrishnan G and Devaki T: Modulatory effect of hesperidin on benzo(a)pyrene induced experimental lung carcinogenesis with reference to COX-2, MMP-2 and MMP-9. Eur J Pharmacol 649: 320-327, 2010.
42. Yumnam S, Park HS, Kim MK, Nagappan A, Hong GE, Lee HJ, Lee WS, Kim EH, Cho JH, Shin SC and Kim GS: Hesperidin induces paraptosis like cell death in hepatoblastoma, HepG2 Cells: Involvement of ERK1/2 MAPK [corrected]. PLoS One 9: e101321, 2014.

43. Birsu Cincin Z, Unlu M, Kiran B, Sinem Bireller E, Baran Y and Cakmakoglu B: Anti-proliferative, apoptotic and signal transduction effects of hesperidin in non-small cell lung cancer cells. Cell Oncol (Dordr) 38: 195-204, 2015.

44. Cincin ZB, Kiran B, Baran Y and Cakmakoglu B: Hesperidin promotes programmed cell death by downregulation of nongenomic estrogen receptor signalling pathway in endometrial cancer cells. Biomed Pharmaco 103: 336-345, 2018.

45. Shaked I, Meerson A, Wolf Y, Avni R, Greenberg D, Gilboa-Geffen A and Soreq H: MicroRNA-132 potentiates cholinergic anti-inflammatory signaling by targeting acetylcholinesterase. Immunity 31: 965-973, 2009.

46. Liu F, Li Y, Jiang R, Nie C, Zeng Z, Zhao N, Huang C, Shao Q, Ding C, Qing C, et al: miR-132 inhibits lipopolysaccharide-induced inflammation in alveolar macrophages by the cholinergic anti-inflammatory pathway. Exp Lung Res 41: 261-269, 2015.

47. Kong H, Yin F, He F, Omran A, Li L, Wu T, Wang Y and Peng J: The effect of miR-132, miR-146a, and miR-155 on MRP8/TLR4-induced astrocyte-related inflammation. J Mol Neurosci 57: 28-37, 2015

This work is licensed under a Creative Commons Attribution-NonCommercial-NoDerivatives 4.0 International (CC BY-NC-ND 4.0) License. 